\title{
UCRL-PROC-232336
}

LAWRENCE LIVERMORE N A T IO N A L LABORATORY

\section{Simulating Electron Effects in Heavy-Ion Accelerators with Solenoid Focusing}

W. M. Sharp, D. P. Grote, R. H. Cohen, A. Friedman, A. W. Molvik, J.-L. Vay, P.A. Seidl, P. K. Roy, J. E. Coleman, I. Haber

June 29, 2007

2007 Particle Accelerator Conference Albuquerque, NM, United States June 25, 2007 through June 29, 2007 
This document was prepared as an account of work sponsored by an agency of the United States Government. Neither the United States Government nor the University of California nor any of their employees, makes any warranty, express or implied, or assumes any legal liability or responsibility for the accuracy, completeness, or usefulness of any information, apparatus, product, or process disclosed, or represents that its use would not infringe privately owned rights. Reference herein to any specific commercial product, process, or service by trade name, trademark, manufacturer, or otherwise, does not necessarily constitute or imply its endorsement, recommendation, or favoring by the United States Government or the University of California. The views and opinions of authors expressed herein do not necessarily state or reflect those of the United States Government or the University of California, and shall not be used for advertising or product endorsement purposes. 


\title{
SIMULATING ELECTRON EFFECTS IN HEAVY-ION ACCELERATORS WITH SOLENOID FOCUSING*
}

\author{
W. M. Sharp, D. P. Grote, R. H. Cohen, A. Friedman, A. W. Molvik - LLNL, Livermore, CA \\ J.-L. Vay, P. A. Seidl, P. K. Roy, J. E. Coleman - LBNL, Berkeley, CA \\ I. Haber - University of Maryland, College Park, MD
}

\begin{abstract}
Contamination from electrons is a concern for solenoid-focused ion accelerators being developed for experiments in high-energy-density physics. These electrons, produced directly by beam ions hitting lattice elements or indirectly by ionization of desorbed neutral gas, can potentially alter the beam dynamics, leading to a time-varying focal spot, increased emittance, halo, and possibly electron-ion instabilities. The electrostatic particle-in-cell code WARP is used to simulate electron-cloud studies on the solenoid-transport experiment (STX) at Lawrence Berkeley National Laboratory. We present self-consistent simulations of several STX configurations and compare the results with experimental data in order to calibrate physics parameters in the model.
\end{abstract}

\section{INTRODUCTION}

The Solenoid Transport Experiment (STX) is an ongoing scaled experiment to study emittance and envelope characteristics of a space-charge-dominated ion beam confined transversely by solenoids [1]. An important aspect of this project is determining how the beam transverse emittance and envelope parameters evolve during solenoid transport, and how these parameters are affected by stray electrons in the system. Results from STX, along with those from the quadrupole-focused High-Current Experiment (HCX), will guide the choice of the transport lattice and electron-control techniques for projected experiments in high-energy-density physics (HEDP) and heavy-ion fusion (HIF).

The STX layout consists of a $300 \mathrm{kV}$ diode producing a $\mathrm{K}+$ beam with a $0.3 \mathrm{~mm}$-mrad emittance, followed by four 2.5-T solenoids, each $51.1 \mathrm{~cm}$ in length and separated by $8.9 \mathrm{~cm}$. A stainless-steel box with intercepting diagnostics is placed after the solenoids to characterize the beam. Negatively biased rings or "traps" are situated at both ends of the solenoids to restrict electron movement toward the source, and an aperture plate may be inserted midway along the upstream trap to reduce beam current by about half. To control the electron flow along the transport lattice, cylindrical electrodes with an adjustable bias voltage are located at the midpoint of each solenoid and between adjacent pairs.

The initial work using the computer code WARP to simulate these experiments, reported in Ref [2], showed qualitative agreement with STX data for early cases with no solenoids and with two. While these simulations did correctly predict the onset of sheath oscillations in the diagnostics region, the time of onset, the frequency spectrum, and the sensitivity to changes in electrode-bias voltages did not accurately replicate the experiment. More recent simulations of the foursolenoid configuration reproduce qualitative features seen in current signals of the STX internal electrodes but do not give comparable integrated charge. Improvements in the numerics, such as the use of mesh refinement near conductors and a greatly reduced time step for electrons have not reduced these discrepancies, so we infer that there remain errors in the physics parameters in WARP or omissions in the physical processes represented.

In this paper, we use recent STX data to guide the choice of several physics parameters in WARP. We first review the physics models currently used in the code and then focus on a particularly clean data set in order to improve the magnetic-field profile in the diagnostics region and to choose more realistic parameters for electron and neutral emission at intercepting diagnostics. We conclude by discussing briefly the direction of future numerical work on this project..

\section{METHOD}

The electrostatic particle-in-cell code WARP [3] has been upgraded to handle multiple species and to model such species interactions as gas desorption, collisional ionization, and release of electrons from conductors [4]. Primary and secondary electron production at walls is managed by the POSINST electron-cloud package [5], while impact ionization is handled by the txPhysics library [6]. An additional module handles desorption of neutrals [7]. The Chombo mesh-refinement code [8] is incorporated into WARP and used in the simulations reported here to give 1-mm resolution in critical region. Electrons are advanced with a smaller time step than the heavier species, as required by the particle Courant condition, and a "drift-Lorentz" electron-advance algorithm [9] allows their gyrofrequency to be ignored when choosing the time step. Various other physical processes, such as recombination, charge exchange, ionization of background gas, and angular scattering, are not yet modeled by WARP but are expected to be inconsequential here.

The simulations here model the beam from the thermionic source to an intercepting target plate in the 
diagnostics package, $2.9 \mathrm{~m}$ from the source. The beam is transported through a $4.6-\mathrm{cm}$-radius beam pipe and focused transversely by fields calculated for ideal solenoids. The lattice alignment, the solenoid fields, and the emitting surface are all assumed to be perfect. The particular layout modeled here for calibrating WARP has a pair of $15-\mathrm{cm}$-square deflecting plate, centered on the STX axis $2 \mathrm{~cm}$ in front of the target plate and separated by $8.5 \mathrm{~cm}$ vertically. The bottom plate is grounded, and the upper plate had a positive bias up to $10 \mathrm{kV}$. The current from the positive deflector plate through a $50 \Omega$ resistor to ground is an important experimental indicator of electron flow near the target plate. Fig. 1 shows this current for a $5-\mathrm{kV}$ bias and two target-plate materials, stainless-steel and copper. Several features of these waveforms are easily understood. The small positive peak at about $2.8 \mu$ s is the "capacitive" current needed to keep the deflector plates at their bias potential as the beam passes between them. This feature is followed by a negative spike, 400 to $500 \mathrm{~mA}$ in magnitude, marking the arrival of the first electrons at the deflector plate. High current at the beam head, formed by sweeping up slower ions, partly accounts for this spike, and some further sharpening may result from transient electric stress as the beam head reaches the grounded target plate. Immediately after the initial electron spike, there is a current trough that we believe is a good indicator of electron emission in the beam body. The electron current then increases in magnitude until the end of the pulse. We attribute this increase to a growing number of electron produce by beam ionization of desorbed neutrals, mainly $\mathrm{H}_{2}$

The high-frequency dips seen in Fig. 1 later in the

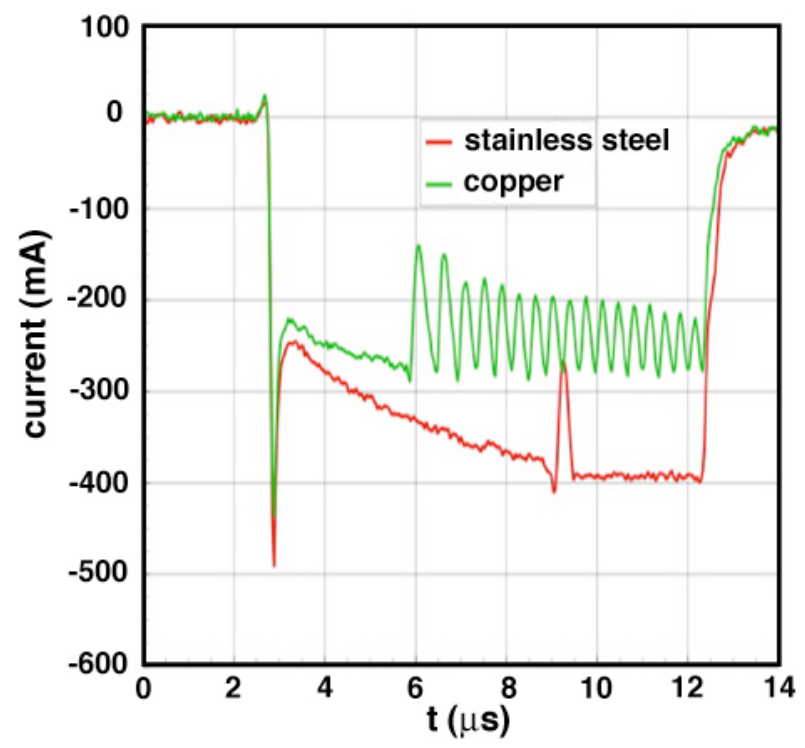

Figure 1: Current through $50 \Omega$ to ground from a positively biased deflector plate using a stainless-steel target plate (red) and a copper one (green). A 5-kV bias was used here, and similar results were found for higher voltages. two current waveforms probably result from sheath oscillations at the deflector plate, but we ignore them here. Also, we defer modeling the data for the copper plate, since most STX data was obtained for a stainlesssteel target.

\section{RESULTS}

\section{$B_{z}$ profile}

Since the STX solenoids are pulsed, the changing $B_{z}$ is expected to induce eddy currents in the surrounding metal structure that will modify the net magnetic field. Measurements last year on a test stand consisting of an STX solenoid with a stainless-steel plate at one end suggest that these eddy currents should reduce the onaxis $B_{z}$ field by more than $40 \%$ in the diagnostics region [10]. The field reduction was found to be smooth and to occur over a distance of about $20 \mathrm{~cm}$ centered at the plate. The actual field reduction on STX has not measured, due to the limited access to the diagnostics region.

The initial WARP simulations of this STX layout, using ideal solenoid fields, showed less than $-20 \mathrm{~mA}$ electron current on the upper deflector plate, shown by a blue curve in Fig. 2. Instead of being collected on the positive plate, most electrons from the target passed between the deflecting plates and were collected on a positively biased cylindrical electrode $13 \mathrm{~cm}$ upstream. Analysis of these simulations shows that this unexpected electron dynamics results from the solenoid fringe field of about $0.02 \mathrm{~T}$ between the deflector plates, giving electrons a gyroradius of less than $0.5 \mathrm{~cm}$ and a substantial $E \times B$ drift velocity .

We model the field reduction due to eddy current by multiplying the ideal on-axis solenoid field $B_{z 0}$ by a function that reduces the field by a fraction $f$ in the vicinity of an axial location $z_{\text {mid }}$ :

$$
B_{z}(z)=B_{z 0}(z)\left\{\frac{1-f}{1-\exp \left[\left(z-z_{\text {mid }}\right) / z_{\text {scale }}\right]}+f\right\} .
$$

Here, $z_{\text {scale }}$ is a measure of the falloff scale length. The associated radial component $B_{r}$ is calculated by requiring that the field divergence be zero. In simulations, we choose $z_{\text {mid }}$ to be the wall of the diagnostics box, and $z_{\text {scale }}=2.8 \mathrm{~cm}$ is found to give a falloff similar to that observe on the test stand.

When the fringe field of the final solenoid reduced by $50 \%$ or more using Eq. (1), we see a dramatic increase in the electron current captured by the positively biased deflector plate. WARP results for $f=$ 0.5 and $f=1$, shown respectively by the magenta and red curves in Fig. 2. When we compare WARP electron emission at the target plate with the deflector plate currents in Fig. 2 for either of these "tailored" $B_{z}$ profiles, we find the virtually all the electrons are 


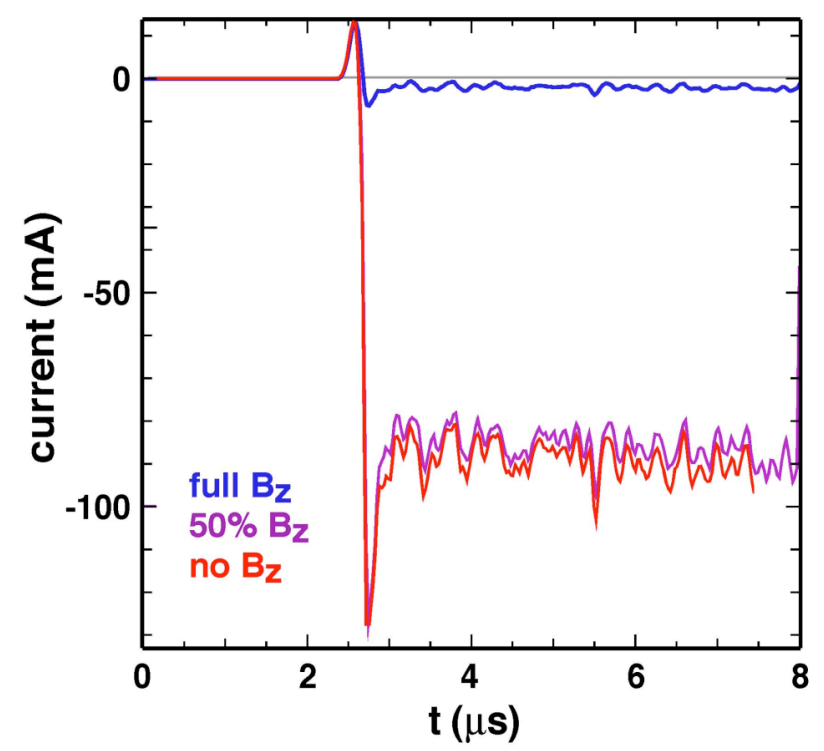

Figure 2: Current from the positive deflector plate for WARP runs using ideal solenoid fields (blue), a field reduced by $50 \%$ near the target plate to account for eddy currents in the STX structure (magenta), and a field

captured by the deflector, and very few secondaries are emitted. In future WARP simulations, we will provisionally use $f=0.5$, since this value is more consistent with the test-stand results.

\section{Electron emission}

The WARP model for electron emission from conductors due to beam-ion impact is a reworking of the POSINST model for secondary electron production $[5,7]$. The model was developed for simulating HCX, which has a $1.8-\mathrm{MeV}$ potassium beam, and it has not been recalibrated for the much lower-energy STX beam. WARP gives a deflector-plate current just after the initial negative spike of about $85 \mathrm{~mA}$ for either profile, compared with the $260 \mathrm{~mA}$ seen in Fig. 1. Since WARP shows that the deflector captures nearly all electrons from the target, we can only replicate the STX data by increasing electron emission by a factor of about three in future simulations.

\section{Neutral desorption}

Recent WARP simulations separate the current due to primary and secondary electrons from that due to ionization electrons. These runs show that the ionization component of the deflector-plate current builds up after the beam hits the target plate to about 20 $\mathrm{mA}$ at $8 \mu \mathrm{s}$. In contrast, the STX result in Fig. 1 shows the electron current increasing by about $100 \mathrm{~mA}$ after the initial negative spike. If we assume that this observed increase is due only to ionization electron, then either the number of neutrals desorbed per ion or the cross section for beam ionization of neutrals is roughly a factor of five too small. Recent work by Kireeff Covo [11] gives us some confidence that the cross section used here is accurate within about $\pm 20 \%$, so we will run future simulations with enhanced desorption.

\section{FUTURE WORK}

The WARP simulations of STX presented here demonstrate the importance of accurately modeling non-ideal experimental details. The $B_{z}$ field reduction due to eddy currents induced in the STX structure by the pulsed solenoid fields is found to alter electron dynamics qualitatively in the diagnostics region. Similarly, the idealized models for electron emission due to beam impact at intercepting diagnostics and for desorption of neutrals are found to seriously underestimate both processes.

With the more realistic field profile and physics parameters found in this work, we expect that future STX simulations will match experimental data better. However, further refinement of the STX model may be needed. The flattening of the deflector-plate current seen in Fig. 1 near the beam tail may indicate that dissociative recombination, not yet implemented in WARP, is reducing the buildup of ionization electrons. Also, the negative total charge collected on certain STX internal electrodes [12] suggests that beam halo is greater than that seen in WARP simulations, possibly due to injector non-uniformities or to lattice misalignments.

\section{ACKNOWLEDGMENTS}

This work was performed under the auspices of the US Department of Energy by the University of California Lawrence Livermore and Lawrence Berkeley National Laboratories under contracts W7405-Eng-48 and DE-AC03-76SF00098.

\section{REFERENCES}

[1] P. A. Seidl, et al., Nucl. Instr. and Meth. A 577, 215 (2007).

[2] W. M. Sharp, et al., Nucl. Instr. and Meth. A 577 , 146 (2007).

[3] D. P. Grote, et al., Nucl. Instr. and Meth. A 415, 428 (1998).

[4] J.-L. Vay, Nucl. Instr. and Meth. A 577, 65 (2007)).

[5] M. A. Furman and M. Pivi, Phys. Rev. ST Accel. Beams 5, 124404 (2002).

[6] http://www.txcorp.com/technologies/TxPhysics/

[7] P. H. Stolz, et al., Phys. Rev. ST Accel. Beams 6, 054701 (2003).

[8] J.-L. Vay, et al., Phys. Plasmas 11, 5, 2928 (2004).

[9] R. H. Cohen, et al., Phys. Plasmas 12, 056708 (2005).

[10] J. Armijo, private communication.

[11] Kireeff Covo, et al., Nucl. Instr. and Meth. A, 577, 139 (2007).

[12] J. E. Coleman, et al., to be submitted to Phys. Rev. ST Accel. Beams (2007). 